\title{
Moderner Sonnenschutz für Kinder und Risikopatienten
}

- Das Lebenszeitrisiko für aktinische Keratose (AK) ist nach Angaben von PD Dr. Günther Hofbauer, Zürich, Schweiz, mit 1:3 sehr hoch. Aus ihr kann sich mit einem Lebenszeitrisiko von 1:20 ein spinozelluläres Karzinom entwickeln. Auch für das Basalzellkarzinom ist das Risiko mit 1:7 hoch, für das maligne Melanom liegt es bei 1:70.

Ein wichtiger externer Faktor bei der Hautkrebsentstehung stellt UV-Strahlung dar, auf die Kinderhaut laut Prof. Dr. Dietrich Abeck, München, besonders empfindlich reagiert, weil die schützende Lichtschwiele noch unzureichend gebildet wird. Daher sollten Kinder Mittagssonne meiden, konsequent textilen Lichtschutz tragen und mit Sonnenschutzmitteln eingecremt werden, die gegen UVA/B schützen und einen hohen Lichtschutzfaktor (LSF) haben. Denn es gelte die Faustregel, dass der reale Schutz auf der Haut nur etwa einem Drittel des angegebenen LSF entspreche, so Abeck.

Nach seiner Erfahrung sind bei vielen Kindern mit perioraler Dermatitis Sonnenblocker mit physikalischem Lichtschutz der Auslöser -insbesondere bei sebostatischem Hauttyp. Diese Cremes würden zu stark okkludieren, so Abeck. Er empfiehlt daher gerne das apo- thekenexklusive Daylong ${ }^{\circledR}$ Kids. Es schützt mit LSF 30 gegen UVA/B, okkludiert aufgrund seiner speziellen Galenik nicht und ist besonders wasserresistent. Produkte der Daylong ${ }^{\circledR}$ _ Serie kosten rund 14 Euro für $100 \mathrm{ml}$. Darüber hinaus gibt es mit Daylong actinica ${ }^{\circledR}$ erstmals ein Medizinprodukt, das sich bei Menschen mit hohem Risiko - beispielsweise Immunsupprimierten-nachweislich zur Prävention der AK eignet [Ulrich C, et al. Br J Dermatol. 2009;161(Suppl 3):78-84]. Hier liegen die Kosten bei rund 20 Euro für $100 \mathrm{ml}$.

pe

Quelle: Satellitensymposium „Brennpunkt Heller Hautkrebs - UV-Strahlung und ihre Wirkung auf die Haut" anlässlich der 14. Jahrestagung der Gesellschaft für Dermopharmazie, Berlin, 22.3.2010 (Veranstalter: Spirig Pharma $\mathrm{GmbH}$ )

\section{Brandwunden: Staphylokokken bekämpfen}

- Wesentliche Ziele der Therapie von Brandwunden sind es, Schmerzen zu lindern und Infektionen zu vermeiden. Die Auswahl des Wundverbandes ist deshalb besonders wichtig. Ein Material, das die analgetischen und antiinfektiven Anforderungen erfüllt, ist der polyhexanidhaltige Feuchtverband Suprasorb ${ }^{\circledR} \mathrm{X}+\mathrm{PHMB}$. Infektionen reduzieren laut einer Mitteilung der Herstellerfirma Lohmann \& Rauscher die Chance auf komplette Wundheilung von 94 auf unter $20 \%$. Das mikrobizide Spektrum von Suprasorb ${ }^{\circledR} X$ + PHMB umfasst auch Methicillin-resistenten Staphylococcus aureus und Vancomycin-resistente Enterokokkenstämme. In-vitro-Untersuchungen hätten gezeigt, dass die Wundauflage eine MRSA-Keimlast von 105 koloniebildenden Einheiten innerhalb von 30 Minuten um 99,9\% reduziert, so der Hersteller.

red

Nach Informationen von Lohmann \& Rauscher

\section{Techniken der Glättung von Glabellafalten}

— Das Botulinum Typ A-Präparat (BoNT-A, Azzalure $^{\circledR}$ ) ist eine neue Behandlungsmöglichkeit zur Glättung von Glabellafalten. Eine Behandlung ist durchschnittlich nur zweimal jährlich notwendig.

Die am häufigsten behandelten Muskeln im oberen Gesichtsdrittel sind der M. corrugator oberhalb der medialen Augenbraue und der M. procerus zwischen der medialen Augenbraue. Die Entspannung dieser Muskeln mit BoNT-A führt zur Hautglättung in diesem Bereich. Die empfohlene Dosierung beträgt 50 Speywood-Einheiten und pro Injektionspunkt 10 Speywood-Einheiten $(=0,05 \mathrm{ml})$.

Für die Behandlung der Glabellafalte werden intramuskuläre Injektionen an fünf Punkten empfohlen, erläuterte Priv.-Doz. Dr. med. Maurizio Podda, Darmstadt: eine Injektion in den M. procerus und zwei in jeden M. corrugator. Die anatomischen Injektionspunkte sind individuell verschieden und sollten vor der Behandlung durch eine Hautmarkierung gekennzeichnet werden.

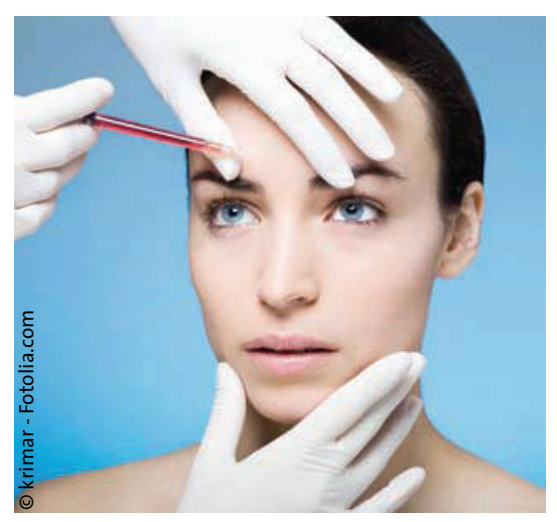

Mit Injektionen an fünf Punkten können die Zornesfalten geglättet werden.
Zur Injektionstechnik sagte Podda, dass die Injektion mit aufwärts gerichteter Kanüle vorgenommen werden sollte, um einen Kontakt mit dem Periost zu vermeiden. Es sollte etwa $5 \mathrm{~mm}$ tief injiziert werden.

Nach der Behandlung kann durch Kühlen und Kompression der injizierten Bereiche eine schnelle Abschwellung erreicht und gleichzeitig das Risiko einer Hämatombildung verringert werden. Die Patienten sollten nach der Injektion die Stirnregion nicht massieren. Eine Wiedervorstellung innerhalb der nächsten 14 Tage ist empfehlenswert, um das Ergebnis beurteilen zu können.

RHA

Wokshop: „Individuelle Behandlungskonzepte mit Botulinumtoxin Typ A“ 27.2.2010 Frankfurt (Veranstalter: Galderma). 